\title{
First radio burst imaging observation from Mingantu Ultrawide Spectral Radioheliograph
}

\author{
Yihua Yan, Linjie Chen, Sijie Yu and CSRH Team $\dagger$ \\ Key Laboratory of Solar Activity, Chinese Academy of Sciences, Beijing, 100012, China \\ email: yyh@nao.cas.cn
}

\begin{abstract}
The Chinese Spectral Radioheliograph (CSRH) with two arrays in $400 \mathrm{MHz}-2 \mathrm{GHz}$ $/ 2-15 \mathrm{GHz}$ ranges with 64/520 frequency channels have been established in Mingantu Observing Station, Inner Mongolia of China, since 2013 and is in test observations now. CSRH is renamed as Mingantu Ultrawide SpEctral Radioheliograph (MUSER) after its accomplishment. We introduce the progress and current status of MUSER. The first burst imaging results of MUSER is presented.
\end{abstract}

Keywords. Radioheliograph, solar radio bursts, flares, CMEs, coronal magnetic field

\section{Introduction}

RHESSI observations indicate that non-thermal particles may account for a significant fraction of flare energy released during initial phase (Lin et al. 2008). Solar eruptions such as flares, CMEs and solar energetic particles etc., may produce radio bursts promptly. The bursts may start around decimetric regime and quickly expand to cover the ultrawide electromagnetism spectrum from a few tens $\mathrm{KHz}$ to microwaves up to mm-wavelengths in a short time scale (cf., Fig. 1 in Benz 2009). Imaging spectroscopy over centimeter and decimetric wavelengths is important for addressing the problems of primary energy release, particle acceleration, transportation processes, and the coronal magnetic fields (Bastian, et al. 1998, Gary \& Keller 2004, Aschwanden 2004, Pick \& Vilmer 2008, Klein et al. 2008, Tomczyk et al. 2013, Nakariakov et al. 2014, 2015, Pohjolainen et al. 2015).

It has been inferred from statistical study of radio dynamic spectral observations that the acceleration site is located in a low-density region corresponding to the plasma frequency of a few hundred $\mathrm{MHz}$, from where electron beams are accelerated in upward (type III) and downward (RS bursts) directions (Aschwanden \& Benz 1997). However, the available radio imaging observations are presently only at a few discrete frequencies in the range $40-150 \mathrm{MHz}$ from Gauribidanur Radioheliograph (Ramesh et al. 1998), in the range 150 - $450 \mathrm{MHz}$ from Nancay Radioheliograph (Radioheliograph Group, 1989), at $5.7 \mathrm{GHz}$ from Siberian Solar Radio Telescope (Grechnev et al. 2004), and at 17/34 GHz from Nobeyama Radioheliograph (Nakajima et al. 1994).

The general purpose instruments including Very Large Array (Napier et al. 1983) and its upgraded version have been used for solar studies (e.g., Chen et al. 2013). But there is only limited time allocation of about $2 \%$ available for solar observations as they were built primarily for non-solar use. Yet their field of view is normally too small to cover the whole solar disk. Therefore it is expected to have a new instrument that is capable of true imaging spectroscopy, with high temporal, spatial, and spectral resolution

$\dagger$ CSRH Team's major members include Drs. Wei Wang, Fei Liu, Zhijun Chen, Lihong Geng, and Jian Zhang etc. 
Table 1. MUSER specifications (cf. Yan et al. 2013)

\begin{tabular}{|c|c|c|}
\hline Frequency range & & $400 \mathrm{MHz} \sim 15 \mathrm{GHz}(\lambda: 75 \sim 2 \mathrm{~cm})$ \\
\hline Frequency resolution & $\begin{array}{c}64 \\
520\end{array}$ & channels for MUSER-I in $0.4 \sim 2 \mathrm{GHz}$ \\
\hline Spatial resolution: & & $\begin{array}{l}\text { I: } 10^{\prime \prime} \sim 50^{\prime \prime} \\
\text { II: } 1.3^{\prime \prime} \sim 10^{\prime \prime}\end{array}$ \\
\hline Temporal resolution: & & I: $25 \mathrm{~ms}$ \\
\hline Dynamic Range & & $25 \mathrm{db}$ (snapshot) \\
\hline Polarizations & & Dual circular L, $\mathrm{R}$ \\
\hline Array & & $\begin{array}{l}\text { I: } 40 \times \phi 4.5 \mathrm{~m} \text { parabolic antennas } \\
\text { II: } 60 \times \phi 2 \mathrm{~m} \text { parabolic antennas }\end{array}$ \\
\hline Maximum baseline & & $>3 \mathrm{~km}$ \\
\hline Single dish beam (Field of view) & & $0.6^{\circ} \sim 7^{\circ}$ \\
\hline
\end{tabular}

(Hudson \& Vilmer 2007, Pick \& Vilmer 2008). The Chinese Spectral Radioheliograph (CSRH, Yan et al. 2004, 2009, 2013) and Frequency-Agile Solar Radiotelescope (FASR, Bastian 2003, Gary 2003) have been proposed to reach this goal. FASR has not been granted for construction yet.

CSRH has been supported as National Major Scientific Research Facility Program of China. The whole budget for the instruments of CSRH project was approved in 2009 . The site survey for the CSRH array was completed at Mingantu town in Inner Mongolia of China and the radio quiet zone protection with $10 \mathrm{~km}$ radius centered at CSRH site was setup in 2008. The construction also started in the autumn of 2008. The whole CSRH system is finished by the end of 2013 and is in commission observations now. CSRH is renamed as Mingantu Ultrawide Spectral Radioheliograph (MUSER) after its accomplishment.

\section{Description of MUSER}

MUSER in centimetric-decimetric wave range is a solar-dedicated radio interferometric array that will be used to carry out imaging spectroscopy of the Sun, to produce high spatial resolution, high time resolution and high frequency resolution images of the Sun simultaneously. The updated main specifications of MUSER are listed in Table 1 (Yan et al. 2013, Wang et al. 2013).

The MUSER frequency range is $0.4-15 \mathrm{GHz}$ which is divided into MUSER-I covering 0.4-2GHz band and MUSER-II covering 2-15GHz band. The MUSER-I contains 40 antennas of $4.5 \mathrm{~m}$ diameter, and the MUSER-II contains 60 antennas of $2 \mathrm{~m}$ diameter. The whole 100 antennas of both MUSER arrays are located on 3 log-spiral arms, and the maximum baseline length is $3 \mathrm{~km}$. Additionally two $20 \mathrm{~m}$ antennas for interferometry experimental in 400-1000 MHz were established in 2011 in MUSER site, which may be incorporated into MUSER-I calibrations later. The solar radio emission in $0.4-2 \mathrm{GHz}$ is detected by each MUSER-I antenna with the broadband feed and outdoor devices including LNA and optic transmitter. The signal with $400 \mathrm{MHz}$ bandwidth, which covers the whole $1.6 \mathrm{GHz}$ bandwidth by scanning 4 times, is then transmitted through optic fibers to indoor devices including optic receiver and analogous receivers with an output in $50-450 \mathrm{MHz}$ range. It is then followed by digital receiver with 1 Gsps A/D converter to receive $400 \mathrm{MHz}$ analogous signal and the digital receiver outputs 16 channels simultaneously for the complex correlations with 2-25 $\mathrm{MHz}$ bandwidth for each channel. The time delay compensation and fringe stopping are considered in the digital correlations. The whole correlation procedure is controlled by a monitoring subsystem. Similar procedure applies to MUSER-II except that the solar radio emission in $2-15 \mathrm{GHz}$ is detected by each MUSER-II antenna with the broadband feed and the IF signal with $400 \mathrm{MHz}$ 


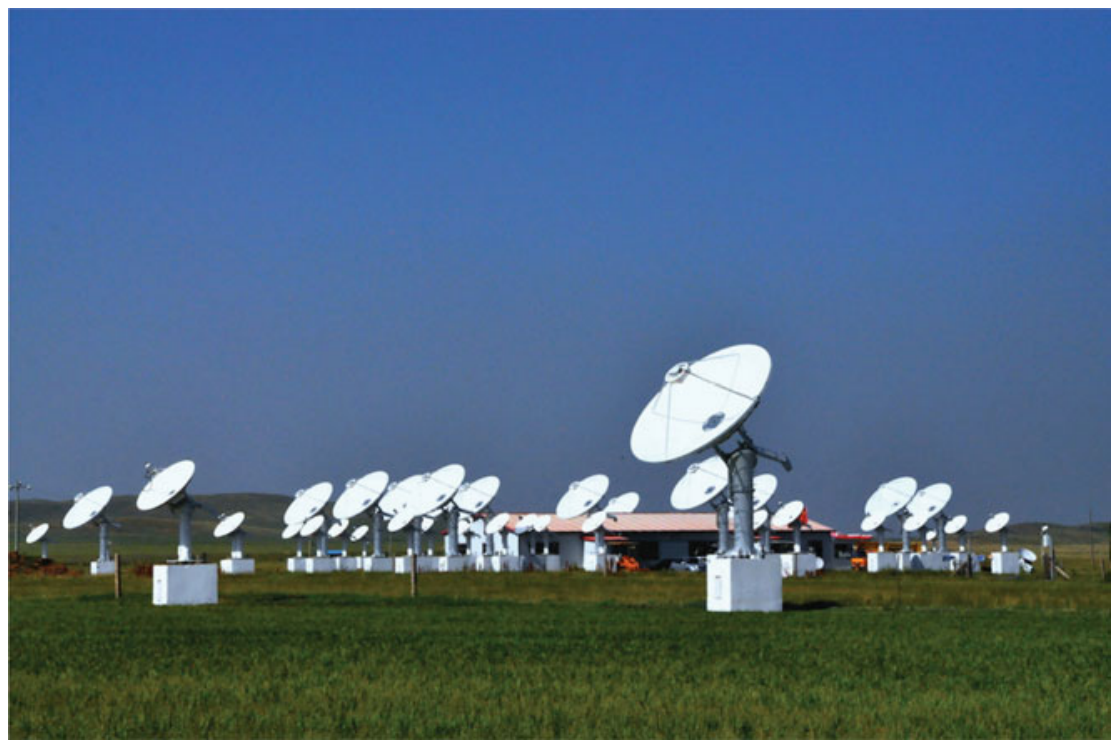

Figure 1. Central part of MUSER-I and MUSER-II arrays.

bandwidth will need to scan the whole $13 \mathrm{GHz}$ bandwidth by 33 times now. Figure 1 shows the central part of the MUSER arrays.

Among the technical issues for MUSER system based on aperture synthesis technique a key problem is that the high performance ultra-wide band feed is needed for reflector antennas. Such feed should be with wide impedance bandwidth, low profile, symmetrical radiation patterns, and fixed phase center over whole band. Solar radio bursts and accordingly their polarizations vary quickly. Therefore, the isolation is important for correct observations of the polarizations. We have successfully developed the feeds for MUSER with VSWR less than 1.5 over most the frequency range and exhibits low profile, ultrawide impedance bandwidth and good radiation characteristics. It is obtained that in nearly $90 \%$ frequency band the polarization degree is less than $5 \%$, which indicates good isolation performance ( $\mathrm{Li}$ et al. 2015a,b). MUSER has been put into test observations and fixed time delays among MUSER-I array have been measured for calibration. The measured RMS variations for MUSER-I were $<1 \mathrm{~ns}$ which is very robust, as similar results obtained after multiple measurements pursued 1 years apart (Liu et al. 2013). For the test observations, fringes of the satellite signals, the Sun, and Cygnus A have been obtained correctly for all baselines. The residuals of the phase closures for every tri-antenna composition were about 2 degrees for both geostationary and GPS satellites (Wang et al. 2013). Whereas the residuals of the phase closures for every tri-antenna composition of MUSER-II were about 1 degree for geostationary satellites. These experiments verify the system design and demonstrate the system performance. The two $20 \mathrm{~m}$ antennas in 400-1000 MHz will be incorporated into MUSER-I calibrations. By simulating the cases when observing the quiet Sun, active regions and the bursts, it is found that shortest baselines for MUSRE-I are important in quiet Sun image recovering (Du et al. 2015).

\section{A Radio Burst Event on 11 November 2014}

On 11 Nov 2014, a radio burst event was registered by MUSER-I array at 400MHz$2 \mathrm{GHz}$ waveband. According to SGD event list there was a C-class flare starting from 04:22 


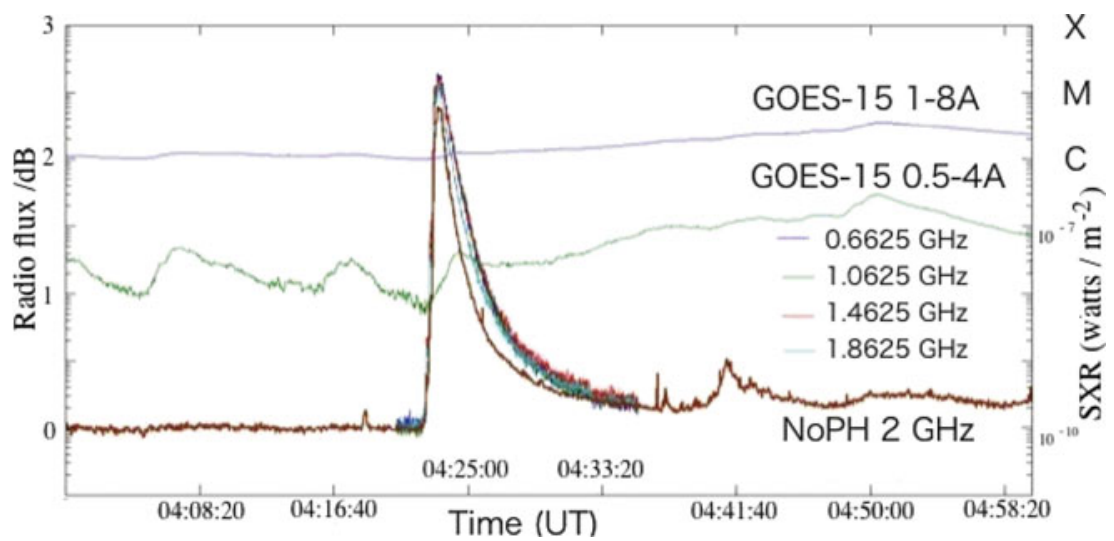

Figure 2. Temporal profiles of GOES SXR flux, the NoPH radio flux at $2 \mathrm{GHz}$ and MUSER-I radio fluxes at $0.6625,1.0625,1.4625$ and $1.8625 \mathrm{GHz}$. The SXR flux is in watts $/ \mathrm{m}^{2}$ unit as marked in the right column whereas the radio flux is in relative unit as marked in the left column.
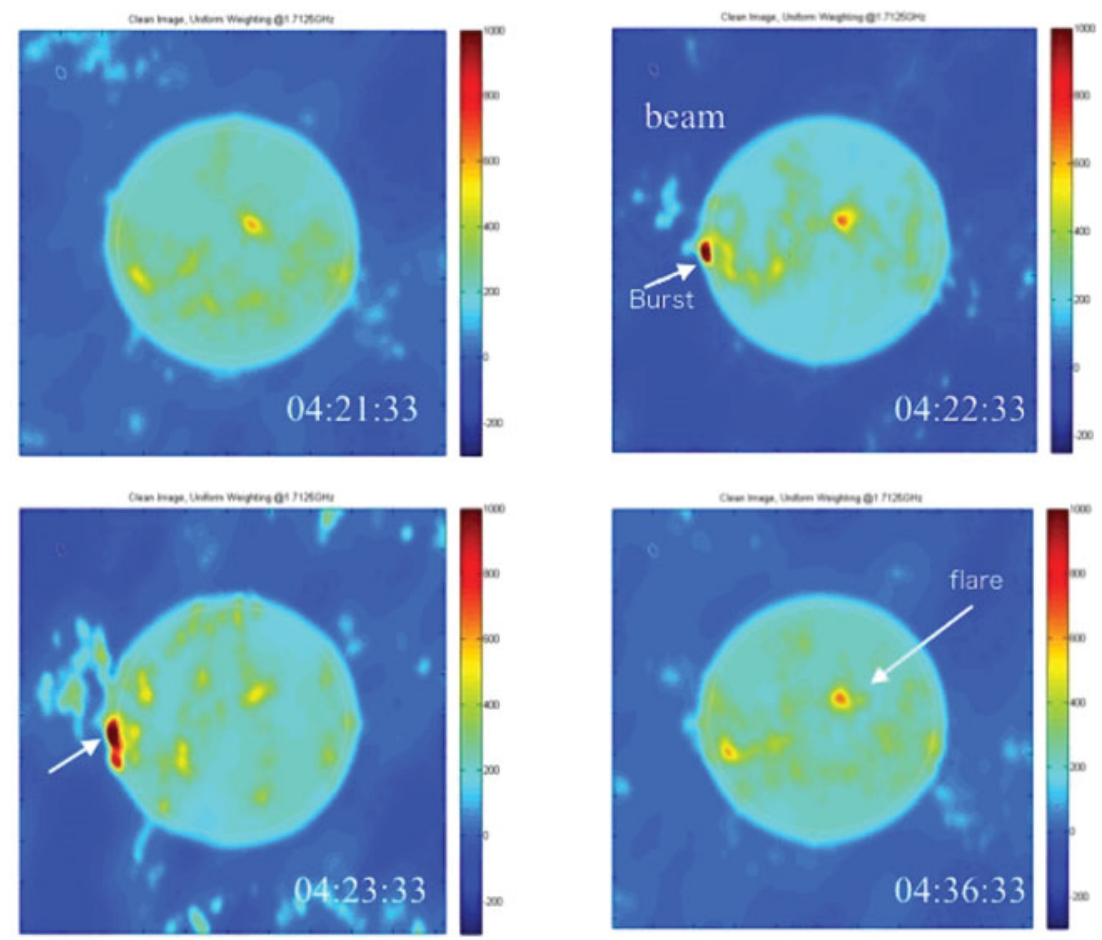

Figure 3. The radio images obtained at 04:21:33 UT (before), 04:22:33 UT (during), 04:23:33 UT (around peak) and 04:36:33 UT (after) the decimetric radio burst.

UT and peaked at 04:49UT in the disk center (/http://www.swpc.noaa.gov/products/ goes-x-ray-flux) AIA/SDO also observed the flare. There were radio bursts during 04:22-04:24UT peaked around 04:23 UT which was attributed to this flare according to SGD. The Nobeyama Radio Polarimeters (NoPH) also demonstrated strong burst signals at 1,2, and $3.75 \mathrm{GHz}$ during 04:22-04:24 UT peaked around 04:23 UT but weak at higher frequencies during the period. So NoRH (Nakajima et al. 1994) only registered the small burst around the flare peak at 04:49 UT. SOHO/LASCO (Brueckner et al. 1995) 

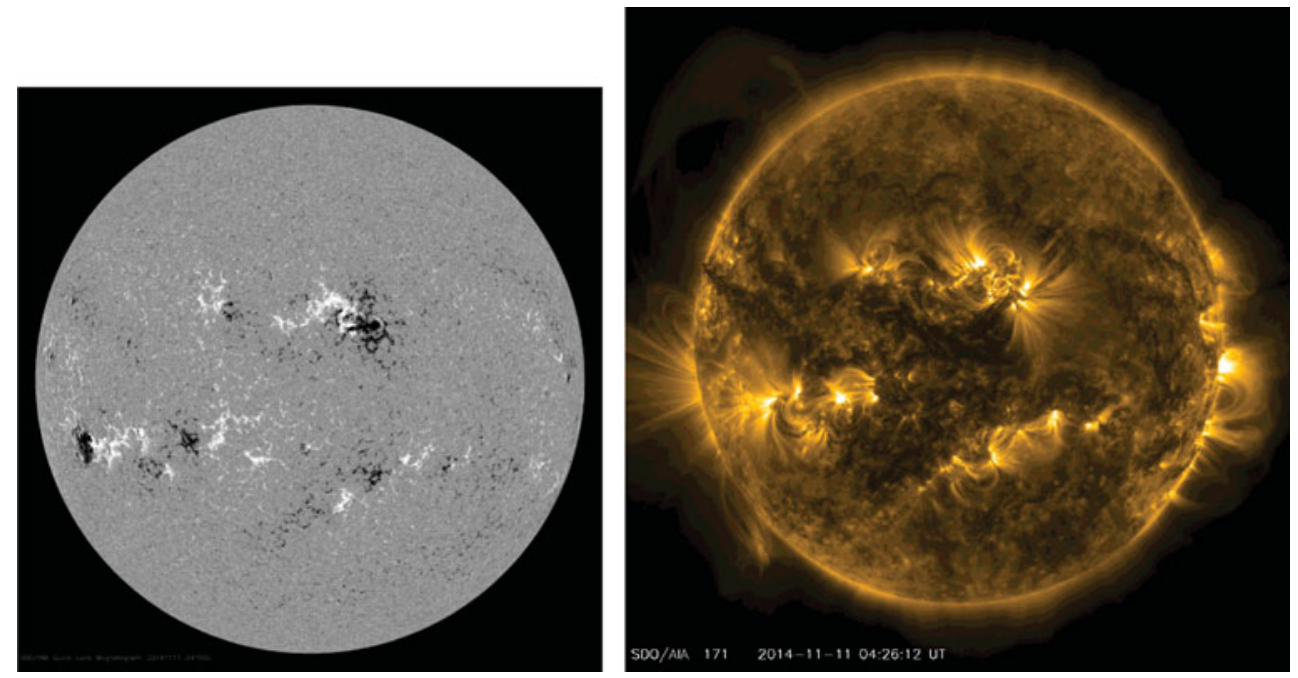

Figure 4. The HMI/SDO magnetogram at 04:15 UT and the AIA/SDO EUV $171 \AA$ image showing the full disk structure.
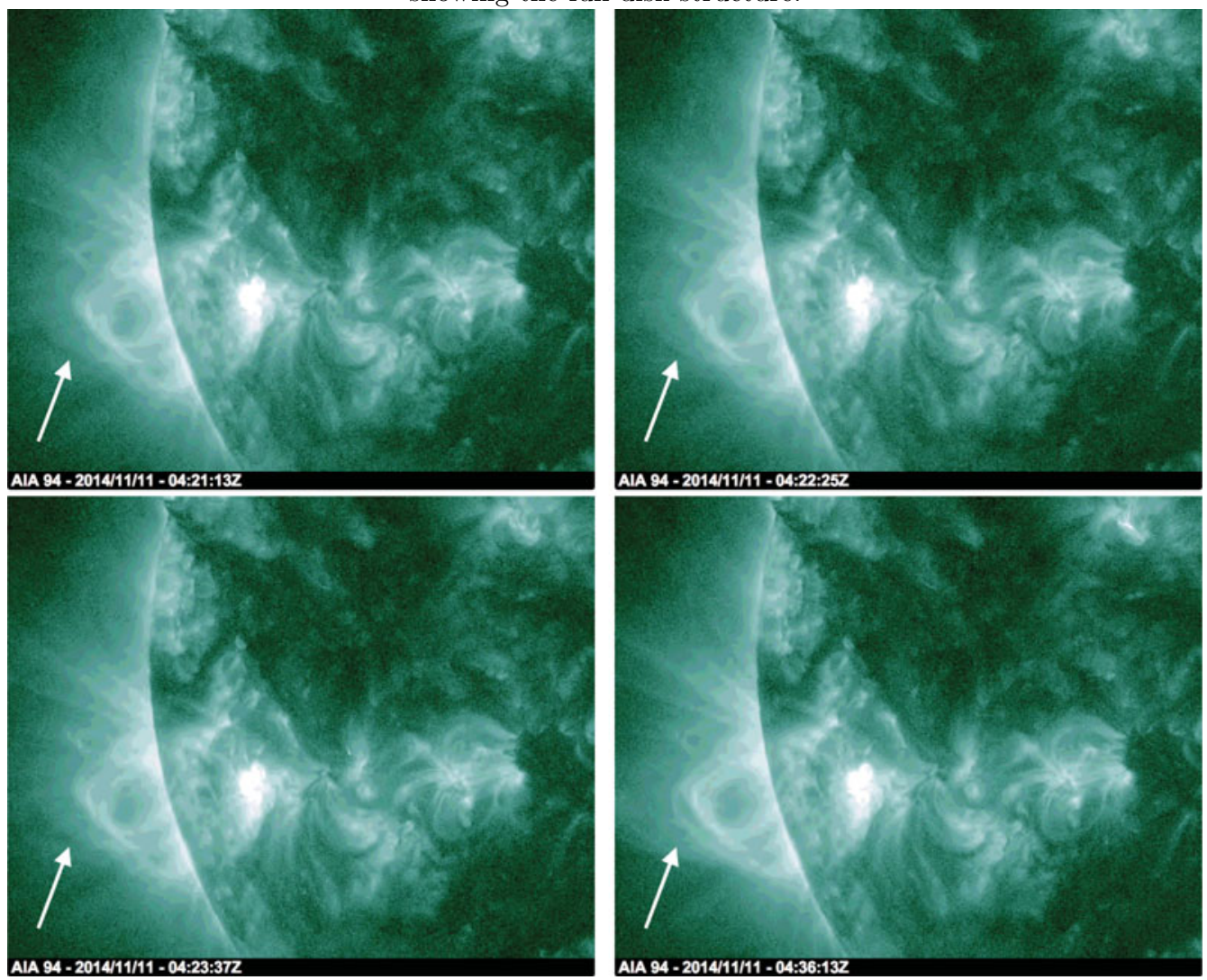

Figure 5. The EUV images obtained at $94 \AA$ showing the eruptive feature of loop opening as indicated by arrow.

registered a CME event with the signature starting around 04:36 UT. So the general scenario would be that the radio bursts during the rising phase would be explained as 

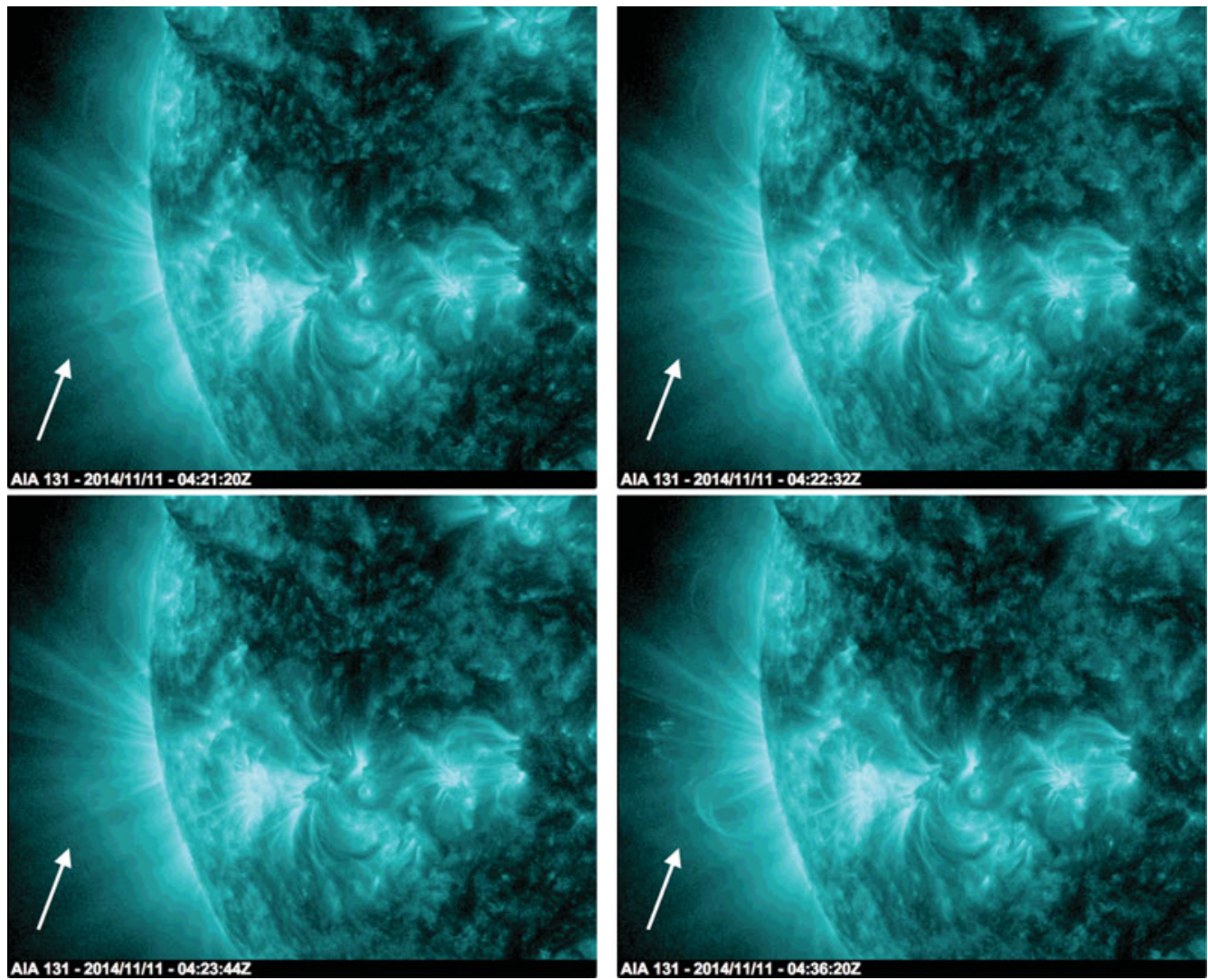

Figure 6. The EUV images obtained at $131 \AA$ showing the eruptive feature of loop opening as indicated by arrow.

the non-thermal process during the impulsive phase to heat the plasma until the flare peak is achieved. Figure 2 shows the comparison of time profiles of GOES SXR flux, the $\mathrm{NoPH}$ radio flux at $2 \mathrm{GHz}$ and MUSER-I radio fluxes at several frequencies spreading over the $0.4-2 \mathrm{GHz}$ band.

However, the radio images obtained from MUSER-I at $1.7 \mathrm{GHz}$ show that the radio burst source is located at the east limb of the Sun, not in the solar disk area. Figure 3 shows the radio images obtained at four instants before, during and after the decimetric radio burst. In order to verify that the radio sources are real, we checked AIA/SDO (Lemen et al. 2012) images at the same time in that location. It is found that there were indeed loop opening at $94 \AA$ and $131 \AA$ corresponding to hot plasmas whereas responses at other AIA/SDO wavelengths were not so obvious. Figure 4 show the HMI/SDO magnetogram (Scherrer et al. 2012) and EUV images at $171 \AA$. It can be seen that the radio image structures agree with the SDO observations. Figure 5 shows the EUV images at $94 \AA$ and Figure 6 shows the EUV images at $131 \AA$ during the eruptive process. Figure 7 shows the images from LASCO $\mathrm{C} 2 / \mathrm{SOHO}$ which indicate the CME at that location.

As there was no radio burst at higher frequencies at the solar east limb, we propose that the decimetric radio burst starting at 04:22 UT in the east limb was due to the solar eruptive events happened in the backside of the Sun but close to the east limb. As radio bursts occurred at higher altitude therefore they were observed by MUSER-I in the earth 

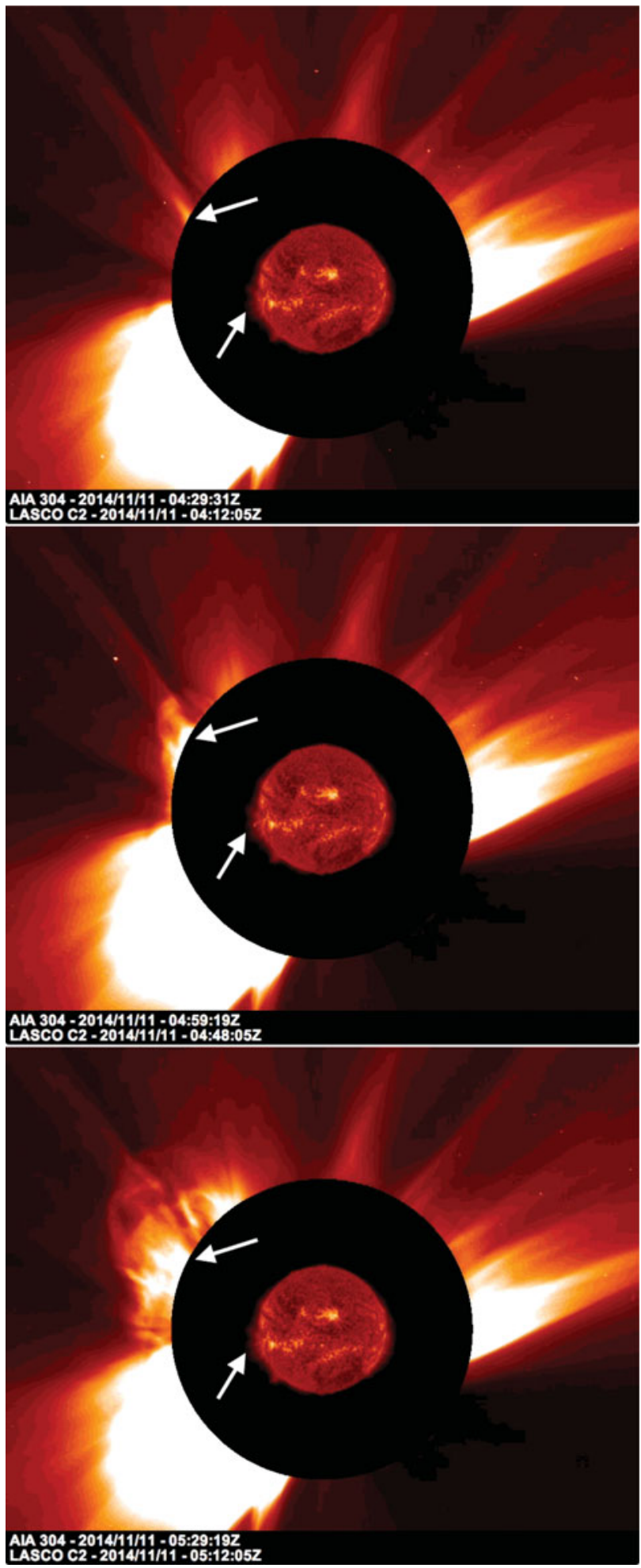

Figure 7. The SOHO/LASCO C2 images showing the structures just before the CME and the starting of the CME. 
direction. Its occurrence during the impulsive phase of the C-class flare in the solar disk was just a coincidence and there should be no physical connection. This demonstrate the importance of the image spectroscopy observation of the solar radio burst.

\section{Concluding Remarks}

In summary, MUSER has the following merits: unique high resolution of time, space and frequency simultaneously over a wide wavelength range; innovation high performance ultra-wide-speed, large-scale digital correlation receiver implemented multi-frequency and snap-shot observations; and new technologies such as remote antenna and wide-band analog signal transmission through optical fibers. MUSERÕs capability of acquiring high temporal, spatial, and spectral resolution over a wide frequency range simultaneously will provide a new observational window on solar activities, such as flares and CMEs at radio wavelength. MUSER yields measuring and imaging of magnetic fields from solar chromosphere to higher corona, helping us to understand the physics of various solar activities and the basic drivers of space weather. MUSER as the new-generation radioheliograph, will be the major solar-dedicated leading radio facility in the world for solar physics.

\section{Acknowledgement}

The CSRH is supported by National Major Scientific Research Facility Program of China with the grant Number ZDYZ2009-3. The NFSC Grants Nos.11433006,11221063, 10921303, the CAS Pilot-B Project XDB09000000, and MOST Grant No.2011CB811401 are acknowledged.

\section{References}

Aschwanden, M. J. \& Benz, A. O. 1997, ApJ, 480, 825

Aschwanden, M. J. 2005, Physics of the Solar Corona, Springer, Berlin, 2005.

Bastian, T. S., Benz, A. O., \& Gary, D. E. 1998, ARAA, 36, 131

Bastian, T. S. 2003, Proc. SPIE, 4853, 98

Benz, A. O. 2009, $\S 4.1 .2 .8$ in Landolt-Bornstein - Group VI Astronomy and Astrophysics Numerical Data and Functional Relationships in Science and Technology, vol. 4B, edited by J.E. Trumper, 1

Brueckner, G. E., Howard, R. A., Koomen, M. J., Korendyke, C. M., Michels, D. J., Moses, J. D., Socker, D. G., Dere, K. P., Lamy, P. L., Llebaria, A., Bout, M. V., Schwenn, R., Simnett, G. M., Bedford, D. K., \& and Eyles, C. J.: 1995, Solar Phys. 162, 357.

Chen, Bin, Bastian, T. S., White, S. M., Gary, D. E., Perley, R., Rupen, M., \& Carlson, B. 2013, ApJL, 763, L21

Du, Jing; Yan, Yihua; Wang, Wei; Liu, Donghao , 2015, PASA, 32, 24

Gary, D. E. 2003, J KAS, 36, 135

Gary, D. E. \& Keller, C. U. 2004, Solar and space weather radiophysics, Kluwer, Dordrecht, 2004

Grechnev, V. V., Lesovoi, S. V., \& Smolkov, G. Ya., et al. 2003, SolPhys, 216, 239

Hudson, H. \& Vilmer, N. 2007, Lect Notes Phys, 725, 81

Lemen, J. R., Title, A. M., Akin, D. J., Boerner, P. F., Chou, C., Drake, J. F., Duncan, D. W., Edwards, C. G., Friedlaender, F. M., Heyman, G. F., Hurlburt, N. E., Katz, N. L., Kushner, G. D., Levay, M., Lindgren, R. W., Mathur, D. P., McFeaters, E. L., Mitchell, S., Rehse, R. A., Schrijver, C. J., Springer, L. A., Stern, R. A., Tarbell, T. D., Wuelser, J.-P., Wolfson, C. J., Yanari, C., Bookbinder, J. A., Cheimets, P. N., Caldwell, D., Deluca, E. E., Gates, R., Golub, L., Park, S., Podgorski, W. A., Bush, R. I., Scherrer, P. H., Gummin, M. A., Smith, P., Auker, G., Jerram, P., Pool, P., Soufli, R., Windt, D. L., Beardsley, S., Clapp, M., Lang, J., \& and Waltham, N.: 2012, Solar Phys. 275, 17.

Li Sha, Yi-hua Yan, Zhi-jun Chen, Wei Wang and Dong-hao Liu, 2015a, RAA, 
Li Sha, Yi Hua Yan, Zhi Jun Chen, Wei Wang and FuShun Zhang, 2015b,PASA,

LIU Dong-hao, YAN Y-i hua, ZHAO An, WANG Wei, 2013, ACTA ELECTRONICA SINICA, 41,570

Nakajima, H., Nishio, M., \& Enome, S., et al. 1994, Proc. IEEE, 82, 705

Nakariakov, V., Bisi, M. M., Browning, P. K., Maia, D., Kontar, E. P., Oberoi, D., Gallagher, P. T., Cairns, I. H., \& and Ratcliffe, H.: 2015, Advancing Astrophysics with the Square Kilometre Array (AASKA14), 169.

Nakariakov, V. M., Kashapova, L. K., \& and Yan, Y.-H.: 2014, Research in Astronomy and Astrophysics 14, 001.

Napier, J. P., Thompson, A. R., \& Ekers, R. D. 1983, Proc. IEEE, 71, 1295

Olsson, R., Kildal, P. S., \& Weinreb, S. 2006, IEEE Trans Ant Prop, 54, 368

Pick, Monique, Vilmer, Nicole 2008, Astron Astrophys Rev, 16, 1

Pohjolainen, S., Karlický, M., van Driel-Gesztelyi, L., \& and Mandrini, C. H.: 2015, Solar Phys. 290, 1.

Radioheliograph Group 1989, SolPhys, 120, 193

Ramesh, R., Subramanian, K. R., Sundararajan, M. S., \& Sastry, Ch. V. 1998, SolPhys, 181, 439

Scherrer, P. H., Schou, J., Bush, R. I., Kosovichev, A. G., Bogart, R. S., Hoeksema, J. T., Liu, Y., Duvall, T. L., Zhao, J., Title, A. M., Schrijver, C. J., Tarbell, T. D., \& and Tomczyk, S.: 2012, Solar Phys. 275, 207.

Wang W., Yan Y. H., Liu D. H., Chen Z. J., Su C., Liu F., Geng L. H., Chen L. J., and Du J., 2013, PASJ, 65, A18

Yan, Y. H., Wang, W., Liu, F., Geng, L. H., Chen, Z. J., \& Zhang, J. 2013, in Solar and Astrophysical Dynamos and Magnetic Activity, Proc. IAU Symposium, vol. 294, edited by A.G. Kosovichev, E.M. de Gouveia Dal Pino, \& Y. Yan, 489

Yan, Y. H., Zhang, J., Wang, W., Liu, F., Chen, Z. J., \& Ji, G. S.2009, Earth Moon Planet, 104, 97

Yan, Y. H., Zhang, J., \& Huang, G., et al. 2004, Proc. 2004 Asia-Pacific Radio Science Conference, Qingdao, China, IEEE, Beijing, p. 391 